\section{IVF - auch bei einzigem Kind höhere Risiken}

Bei Schwangerschaften nach In-vitro-Fertilisation (IVF) ist die Rate an Komplikationen durch Mehrlingsschwangerschaften erhöht. Ein Autorenteam prüfte, ob diese Risiken bei Schwangerschaften mit nur einem Kind und SingleEmbryo-Transfer ebenfalls bestehen.

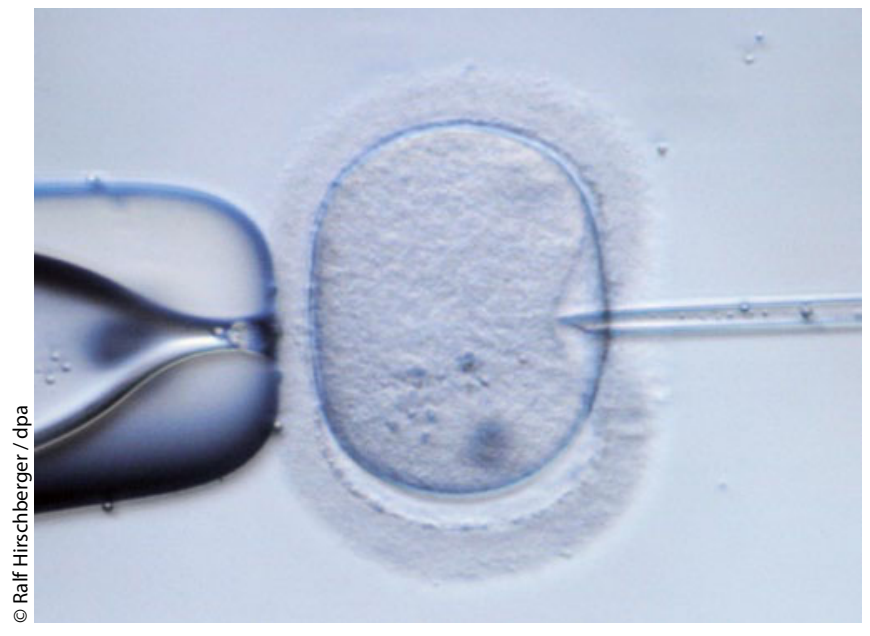

\section{Blinddarm-Op als Kind erhöht Risiko für Infarkt}

Werden Tonsillen oder Blinddarmfortsatz in jungen Jahren operativ entfernt, kann das einer frühzeitigen koronaren Herzkrankheit (KHK) den Weg bereiten. Nach einer schwedischen Fall-Kontroll-Studie steigt das Infarktrisiko nach Tonsillektomie um $44 \%$ und nach Appendektomie um $33 \%$. Für die Studie wurden die Daten von allen zwischen 1955 und 1970 geborenen und vor dem 20. Lebensjahr operierten Schweden ausgewertet. Die Nachbeobachtungszeit lag im Durchschnitt bei 23,5 Jahren. Der Anstieg des Herzinfarktrisikos bei den operierten Personen war in beiden Gruppen signifikant. Die Autoren erklären ihre Beobachtung damit, dass Mandeln und Blinddarm sekundäre Lymphorgane sind. Die Entfernung könne daher die Immunantwort abschwächen. Eine mögliche Folge wäre eine erhöhte inflammatorische Aktivität, welche wiederum die Entstehung einer KHK fördern könne. Im Gegensatz dazu blieb das Herzinfarktrisiko davon unberührt, wenn die Operation erst mit 20 Jahren oder später erfolgte. Dr. Beate Schumacher

Cohen AT et al. Lancet 2008; 371: 387-94

\section{Keine Sorge bei Spätsprechern}

Wenn Kleinkinder in der Sprachentwicklung hinterherhinken, sagt das wenig über ihre weitere Entwicklung: Bis zum Schuleintritt haben die meisten Kinder ihren sprachlichen Rückstand aufgeholt. Auch Verhaltensauffälligkeiten oder psychische Probleme sind bei ihnen einer neuen Studie zufolge nicht häufiger zu erwarten. Rund $10 \%$ der Zweijährigen $(n=142)$ wurden darin als "Spätsprecher" eingestuft. Nach der Einschätzung ihrer Eltern hatten sie häufiger psychische Schwierigkeiten als Kleinkinder mit alterstypischem Wortschatz $(n=1.245)$. So wurden z. B. bei $13 \%$ internalisierende Auffälligkeiten wie Schüchternheit, Traurigkeit und mangelnde Aktivität angegeben, im Vergleich zu $8 \%$ bei den Kontrollkindern. Externalisierende Auffälligkeiten wurden bei $19 \%$ bzw. $13 \%$ beschrieben. Die Unterschiede beschränkten sich jedoch nur auf das Kleinkindalter. In späteren Lebensjahren hatten die Spätsprecher nicht mehr psychische Probleme als die Kinder, die schon früher gesprochen hatten.

Dr. Beate Schumacher

Whitehouse AJO et al. Pediatrics 2011 Jul 4. [Epub ahead of print] 